\title{
Estimation of Setchenow Coefficients of Ammonia in Aqueous Salt Solutions
}

\author{
Masunobu MAEDA*, Osamu HISADA and Kaname ITO
}

Received May 27, 1987 ; Accepted July 13, 1987

\section{INTRODUCTION}

The activity coefficient $y$ and solubility $S$ of a nonelectrolyte in an aqueous salt solution at a low salt concentration have been empirically found to be expressed as a function of the salt concentration $c$ in molar units $(1,2)$.

$$
\log y / y_{0}=\log s_{0} / S=k_{s} c
$$

where $y_{0}$, to which unity is usually assigned, and $S_{0}$ are for a salt-free solution, and the proportionality factor $k^{\prime}$, which concerns the ion-nonelectrolyte interaction, is known as the setchenow (salting) coefficient and has a characteristic value for a given salt-nonelectrolyte pair. A positive value of $k_{s}$ corresponds to salting-out, and if $k_{s}$ is negative, salting-in is observed.

So far, a number of theories have been developed for the prediction of the $k_{s}$ values for nonpolar nonelectrolytes. A comparative tabulation of the various theories of salting-in and -out with their features are given by Conway (3). The scaled particle theory $(4,5)$ among others has been successfully applied to the calculation of solubilities of nonpolar gases such as $\mathrm{H}_{2}, \mathrm{O}_{2}, \mathrm{~N}_{2}$, hydrocarbons, and noble gases in aqueous salt solutions (6-8). On the other hand, as far as we have surveyed the literature, it seems that no attempt has been made to calculate theoretically the setchenow coefficients of polar nonelectrolytes in aqueous salt solutions.

As was reported in previous papers(9-12), we measured the activity coefficients of ammonia in various 1 - 1 electrolyte solutions of different concentrations at $25^{\circ} \mathrm{C}$. In the present work, therefore, the Setchenow coefficients of ammonia in 1 - 1 salt solutions were calculated according to the scaled particle theory, and compared with the experimental values in order to examine the applicability of this theory to polar nonelectrolyte systems.

\section{PROCEDURES FOR THEORETICAL ESTIMATION OF SETCHENOW COEFFICIENTS OF} AMMONIA

Shoor and Gubbins(6) derived from the scaled particle theory the following expression for $k_{s}$ in molar units.

$$
k_{s}=\left[\mathrm{d}\left(\bar{g}_{1} \mathrm{~h} / 2.3 k T\right) / \mathrm{d}(c)\right]_{c \rightarrow 0}+\left[\mathrm{d}\left(\bar{g}_{1}^{\mathrm{s}} / 2.3 k T\right) / \mathrm{d}(c)\right]_{c \rightarrow 0}=k_{\alpha}+k_{\beta}
$$

where $k$ is the Boltzmann constant, $T$ is the temperature in degrees Kelvin, $c$ is the concentration of the salt in molar units, and $\bar{g}_{1} \mathrm{~h}$ is the Gibbs energy when a cavity large enough to accomodate the nonelectrolyte molecule is formed in the solution, and $\bar{g}_{1} s_{\text {is }}$ the Gibbs energy required to place the nonelectrolyte molecule in this cavity in the face of interactions with the water molecules and the ions of the electrolyte. $k_{\alpha}$ and $k_{\beta}$ represent the contributions to the salting coefficient of each of the two terms on the left of Eq.2. The present problem is to derive the expressions for $k_{\alpha}$ and $k_{\beta}$ in terms of parameters characteristic of the nonelectrolyte, the solvent, and the ions of the 1 - 1 salt. In what follows we use subscript 1 to represent the parameters for

Department of Applied Chemistry, Nagoya Institute of Technology(Gokiso-cho, Showa-ku, Nagoya 466)

Key words: Activity Coefficient of Ammonia, Setchenow Coefficient, The Scaled Particle Theory, Salting-In, Salting-out 
the nonelectrolyte (ammonia), 2 those for the solvent(water), 3 those for the cation, and 4 those for the anion.

Expression for $k_{\alpha}$ This expression may be the same as that derived previously by Shoor and Gubbins (6), regardless of whether the nonelectrolyte is polar or nonpolar. According to them, the final expression for $k_{\alpha}$ is given by $\mathrm{Eq} \cdot 3$.

$$
\begin{aligned}
k_{\alpha}= & {\left[\mathrm{d}\left(\bar{g}_{1}^{\mathrm{h}} / 2.3 k T\right) / \mathrm{d}(c)\right]_{c \rightarrow 0}=2.15 \times 10^{20}\left(\sigma_{3}{ }^{3}+\sigma_{4}{ }^{3}\right)-2.47 \times 10^{-4} \mathrm{x} \phi_{0}+} \\
& \sigma_{1}\left[6.45 \times 10^{20}\left(\sigma_{3}{ }^{2}+\sigma_{4}{ }^{2}\right)+1.34 \times 10^{28}\left(\sigma_{3}{ }^{3}+\sigma_{4}^{3}\right)-4.23 \times 10^{4} \phi_{0}\right]+ \\
& \sigma_{1}{ }^{2}\left[6.45 \times 10^{20}\left(\sigma_{3}+\sigma_{4}\right)+4.01 \times 10^{28}\left(\sigma_{3}{ }^{2}+\sigma_{4}{ }^{2}\right)+1.32 \times 10^{36}\left(\sigma_{3}{ }^{3}+\right.\right. \\
& \left.\left.\sigma_{4}{ }^{3}\right)-4.17 \times 10^{12} \phi_{0}\right]
\end{aligned}
$$

where $\sigma^{\prime} \mathrm{s}$ are the diameters of the species in $\mathrm{cm}$ units and $\phi_{0}$ the apparent molal volume of the salt at infinite dilution in $\mathrm{cm}^{3} \mathrm{~mol}^{-1}$ units.

Expression for $k_{B}$ The $k_{\beta}$ term for the polar nonelectrolyte should be different from that ${ }^{\beta}$ for the nohpolar one derived previously $(6)$ according to the Lennard-Jones potential taking account of the ion-induced dipole interaction.

According to shoor and Gubbins (6), $\bar{g}_{1}{ }^{s}$ in a single salt solution is approximated by Eq. 4 .

$$
\bar{g}_{1}{ }^{s}=\sum_{j=2}^{4} \rho_{j} \rho_{\sigma_{1 j}}^{\infty} u_{1 j}(r) 4 \pi r^{2} \mathrm{~d} r
$$

where $\rho_{j}$ is the number density (particles $/ \mathrm{cm}^{3}$ ) of a solution species, $\sigma_{1 j}$ is assumed to be equal to $\left(\sigma_{1}+\sigma_{j}\right) / 2$, and $u_{j j}(r)$ is the pair potential between the nonelectrolyte and species $j$ at a distance of $r$. For the 1 - electrolyte (7)

$$
\rho_{3}=\rho_{4}=N c / 1000
$$

where $N$ is Avogadro's number. The number density of water is given by Eq.6(7).

$$
\rho_{2}=\left(N d_{2} / M_{2}\right)[1-c \phi / 1000]
$$

in which $d_{2}$ is the density of pure water, $M_{2}$ the molecular weight of water, and $\phi$ the apparent molal volume of the salt. The pair potential $u_{j}(r)$ for the interaction between the polar nonelectrolyte(ammonia) and species $j$ was assumed to be expressed in terms of the stockmayer potential(13) as is written by Eqs.7a and $7 \mathrm{~b}, u_{12}(r)$ being derived after averaging the interaction between the permanent dipole of the polar solute and that of the water molecule over all orientations.

$$
\begin{aligned}
& u_{12}(r)=4 \varepsilon_{12}\left[\left(\sigma_{12} / r\right)^{12}-\left(\sigma_{12} / r\right)^{6}\right]-(2 / 3 k T) \mu_{1}{ }^{2} \mu_{2}{ }^{2} r^{-6} \\
& u_{1 j}(r)=4 \varepsilon_{1 j}\left[\left(\sigma_{1 j} / r\right)^{12}-\left(\sigma_{1 j} / r\right)^{6}\right] \text { (for } j=3 \text { and } 4 \text { ) }
\end{aligned}
$$

where $\varepsilon_{1 j}(j=2-4)$ is the mixture potential parameter, which is related to the pure component parameters $\varepsilon_{1}$ and $\varepsilon_{j}$ by the approximate mixing rule (13),

$$
\varepsilon_{1 j}=\left(\varepsilon_{1} \varepsilon_{j}\right)^{1 / 2}
$$

and $\mu_{1}$ and $\mu_{2}$ are the dipole moments of the nonelectrolyte and water molecule, respectively? Substituting the above expressions for $\rho_{2}, \rho_{3}, \rho_{4}, u_{12}(r)$, and $u_{1 j}(r)$ into Eq. 4 and performing the integrations give

$$
\begin{aligned}
\bar{g}_{1}^{s} / 2.3 k T= & -(32 \pi N c / 9000 \times 2.3 k T)\left[\varepsilon_{13} \sigma_{13}^{3} / k+\varepsilon_{14} \sigma_{14}{ }^{3} / k\right]-\left(32 \pi N d_{2} / 9 \times 2.3 \times\right. \\
& \left.k T M_{2}\right)[1-c \phi / 1000] \times \varepsilon_{12} \sigma_{12} / k-\left(8 \pi N d_{2} / 9 \times 2.3 k^{2} T^{2} M^{2}\right)[1- \\
& c \phi / 1000] \mu_{1}{ }^{2} \mu_{2}^{2} / \sigma_{12}{ }^{2}
\end{aligned}
$$


If the derivative of $\mathrm{Eq.9}$ is taken with respect to $c$ and numbers for the various constants $\left(M_{2}=18,02 ; d_{2}=0.997 \mathrm{~g} / \mathrm{cm}^{3} ; N=6.02 \times 10^{23} / \mathrm{mol} ; \mathrm{k}=1.38 \mathrm{x}\right.$ $10^{-16}$ erg $\left./ \mathrm{K}\left(\mathrm{erg}=10^{-7} \mathrm{~J}\right) ; T=298 \mathrm{~K}\right)$ are inserted, the final expression for $k_{B}$ is obtained.

$$
\begin{aligned}
& k_{B}=\left[\mathrm{d}\left(\bar{g}_{1}^{\mathrm{s}} / 2.3 k T / \mathrm{d}(c)\right]_{c \rightarrow 0}=-9.80 \times 10^{18}\left[\varepsilon_{13} \sigma_{13}{ }^{3} / k+\varepsilon_{14} \sigma_{14}{ }^{3} / k\right]+5.43 \mathrm{x}\right. \\
& 10^{17} \phi_{0} \varepsilon_{12} \sigma_{12}{ }^{3} / k+2.39 \times 10^{46} \phi_{0} \mu_{1}{ }^{2} \mu_{2}{ }^{2} / \sigma_{12} \\
& =-1.23 \times 10^{8}\left[\left(\varepsilon_{1} / k\right)^{1 / 2}\left(\varepsilon_{3} / k\right)^{1 / 2}\left(\sigma_{1}+\sigma_{3}\right)^{3}+\left(\varepsilon_{1} / k\right)^{1 / 2}\left(\varepsilon_{4} / k\right)^{1 / 2}\left(\sigma_{1}+\sigma_{4}\right)^{3}\right]+ \\
& 6.79 \times 10^{16} \phi_{0}\left(\varepsilon_{1} / k\right)^{1 / 2}\left(\varepsilon_{2} / k\right)^{1 / 2}\left(\sigma_{1}+\sigma_{2}\right)^{3}+1.91 \times 10^{47} \phi_{0} \mu_{1}^{2} \mu_{2}^{2} / \\
& \left(\sigma_{1}+\sigma_{2}\right)^{3}
\end{aligned}
$$

The setchenow coefficient $k_{s}$ can be calculated by adding $k_{\alpha}(e q \cdot 3)$ and $k_{\beta}(\mathrm{Eq} \cdot 10)$. parameters necessary to calculate $k_{s}$ are the apparent molal volume of the salt at infinite dilution $\left(\phi_{0}\right)$, the diameters for $\mathrm{NH}_{3}\left(\sigma_{1}\right), \mathrm{H}_{2} \mathrm{O}\left(\sigma_{2}\right)$, the cation $\left(\sigma_{3}\right)$, and the anion $\left(\sigma_{4}\right)$, the dipole moments of $\mathrm{NH}_{3}\left(\mu_{1}\right)$ and $\mathrm{H}_{2} \mathrm{O}\left(\mu_{2}\right)$, and the energy parameters for ammonia $\left(\varepsilon_{1} / k\right), \mathrm{H}_{2} \mathrm{O}\left(\varepsilon_{2} / k\right)$, the cation $\left(\varepsilon_{3} / k\right)$, and the anion $\left(\varepsilon_{4} / k\right)$. The apparent molal volumes were calculated from those for ions given by Friedman and Krishnan(14). The diameters for alkali and halide ions given by Waddington(15) were accepted, since it was found(16) that the observed $k$ values for nonpolar nonelectrolytes in alkali halide solutions gave the best agreement with those calculated from the scaled particle theory by simply doubling Waddington's crystallographic radii. As the diameter for $\mathrm{NO}_{3}$ ion was accepted $\sigma_{4}=3.92 \times 10^{-8} \mathrm{~cm}$, which was estimated in such a way that the $k$ values for nonpolar solutes in alkali nitrate solutions calculated from the scaled particle theory fitted to the experimental values(16). The crystal diameter for $\mathrm{ClO}_{4}{ }^{-}$ion $=4.81 \times 10^{-8} \mathrm{~cm}$ was taken from Gmelins Handbuch (17). The parameters for $\mathrm{NH}_{3}\left(\sigma_{1}, \mu_{1}, \varepsilon_{1} / k\right)$ and $\mathrm{H}_{2} \mathrm{O}\left(\sigma_{2}, \mu_{2}, \varepsilon_{2} / k\right)$ for the stockmayer po-

\begin{tabular}{|c|c|c|c|c|c|}
\hline species & $\sigma \times 10^{8} / \mathrm{cm}$ & $\begin{array}{l}\mu \times 10^{18} \\
\text { cgs esu*1) }\end{array}$ & $\alpha \times 10^{24} / \mathrm{cm}^{3}$ & ${ }_{\mathrm{cm}_{0}}^{\prime} 3_{\mathrm{mol}^{-1 * 2)}}$ & $\varepsilon / k / K$ \\
\hline $\mathrm{NH}_{3}$ & 2.60 & 1.47 & & & 320 \\
\hline $\mathrm{H}_{2} \mathrm{O}$ & 2.65 & 1.83 & & & 380 \\
\hline $\mathrm{Li}^{+}$ & 1.48 & & & -6.3 & 5645 \\
\hline $\mathrm{Na}^{+}$ & 2.02 & & & -6.6 & 556 \\
\hline $\mathrm{k}^{+}$ & 2.64 & & & 3.6 & 300 \\
\hline $\mathrm{Cl}^{-}$ & 3.64 & & 3.02 & 23.2 & 218 \\
\hline $\mathrm{Br}^{-}$ & 3.96 & & 4.17 & 30.1 & 302 \\
\hline$I^{-}$ & 4.48 & & 6.28 & 41.6 & 326 \\
\hline $\mathrm{NO}_{3}{ }^{-}$ & 3.92 & & 3.60 & 34.4 & 243 \\
\hline $\mathrm{ClO}_{4}{ }^{-}$ & 4.81 & & 4.16 & 49.5 & 110 \\
\hline
\end{tabular}
tential were from Hirschfelder et al.(13). The parameter values described above are listed in Table 1. The energy parameters for the cations and anions,

Table 1. Parameter values for calculating setchenow coefficients of ammonia

*1) cgs esu $=3.33 \times 10^{-12} \mathrm{Cm} ; * 2$ ) partial molal volumes for ions, from which a $\phi_{0}$ value for a given electrolyte can be calculated by the algebraic summation of the $\phi_{0}$ values for thr component ions. 
which are not available, were estimated as follows. As was pointed out in a previous paper(9), the interaction of ammonia with anions would be weak compared with that with cations. Thus, the energy parameters for the anions were assumed to be estimated from the Mavroyannis-stephen theory (18). According to the theory, the expression for $\varepsilon_{4} / k$ for the anion is given by Eq.11.

$$
\varepsilon_{4} / k=2.28 \times 10^{-8} \alpha_{4}{ }^{3 / 2} z_{4}{ }^{1 / 2} / \sigma_{4}{ }^{6}
$$

where $\alpha_{4}$ is the polarizability of the anion, and $z_{4}$ the total number of electrons in the anion. Ion polarizabilities were taken from Conway (19) for the halide ions, Landort-Börnstein(20) for $\mathrm{NO}_{3}{ }^{-}$ion, and Gmelins Handbuch (17) for $\mathrm{ClO}_{4}$ ion. The values of $\varepsilon_{3} / k$ for the cations ( $\mathrm{Li}^{+}, \mathrm{Na}^{+}$, and $\mathrm{K}^{+}$), which are expected to make strong interactions with ammonia, were then evaluated so as to fit the $k_{s}$ values in LiCl, $\mathrm{NaCl}$, and $\mathrm{KCl}$ solutions predicted from the scaled particle theory to the observed values. The $\varepsilon_{3} / k$ and $\varepsilon_{4} / k$ values thus calculated are given in Table 1 .

\section{RESULTS AND DISCUSSION}

The predicted $k_{s}$ values with the relative contributions of the two terms $k_{\alpha}$ and $k_{\beta}$ to $k_{s}$ are compared with the observed ones in Table 2 .

Table 2. Comparison between observed and calculated Setchenow coefficients $k_{s}$ for ammonia with $k_{\alpha}$ and $k_{\beta}$ values

\begin{tabular}{|c|c|c|c|c|}
\hline \multirow[b]{2}{*}{ salt } & \multirow{2}{*}{$\begin{array}{c}\text { obsd } \\
k_{s}\end{array}$} & \multicolumn{3}{|c|}{ est } \\
\hline & & $k_{s}$ & $k_{\alpha}$ & $\mathbf{k}_{\beta}$ \\
\hline LiCl & $\left.-0.038^{\star} 1\right)$ & $\left.-0.038^{\star} 6\right)$ & 0.095 & -0.133 \\
\hline $\mathrm{NaCl}$ & $0.041^{\star 51}$ & $0.041^{* 61}$ & 0.114 & -0.073 \\
\hline $\mathrm{KCl}$ & $0.060^{* 31}$ & $0.060^{* 61}$ & 0.101 & -0.041 \\
\hline LiBr & $-0.033^{\star 21}$ & -0.040 & 0.097 & -0.137 \\
\hline $\mathrm{NaBr}$ & $0.017^{\star 31}$ & 0.039 & 0.117 & -0.078 \\
\hline $\mathrm{KBr}$ & $0.035^{* 31}$ & 0.058 & 0.105 & -0.047 \\
\hline LiI & $-0.062^{* 21}$ & -0.019 & 0.111 & -0.131 \\
\hline $\mathrm{NaI}$ & $\left.-0.007^{*} 3\right)$ & 0.060 & 0.131 & -0.072 \\
\hline $\mathrm{KI}$ & $0.017^{\star 31}$ & 0.079 & 0.120 & -0.041 \\
\hline LiNO $_{3}$ & $\left.-0.023^{*} 4\right)$ & -0.034 & 0.076 & -0.110 \\
\hline $\mathrm{NaNO}_{3}$ & $\left.0.038^{\star 4}\right)$ & 0.045 & 0.095 & -0.050 \\
\hline $\mathrm{KNO}_{3}$ & $0.060 * 4)$ & 0.064 & 0.083 & -0.019 \\
\hline $\mathrm{LiClO}_{4}$ & $\left.-0.065^{\star 4}\right)$ & 0.068 & 0.125 & -0.058 \\
\hline $\mathrm{NaClO}_{4}$ & $0.003^{\star 4)}$ & 0.147 & 0.144 & 0.002 \\
\hline
\end{tabular}

*1) Unpublished result; *2) from Ref.1; *3) from Ref.2;

*4) from Ref.9; *5) from Ref.12;*6) fitted to the observed $k_{s}$ values in order to estimate the energy parameters for the cations.

It is apparent that $k_{\alpha}$ is positive, while $k_{\beta}$ is negative, irrespective of the types of the salts. An exception is the $\mathrm{NaClO}_{4}$ system. The fact that $k_{\alpha}$ increases with increasing ionic size denotes that it becomes more difficult to 
form a cavity and thus the Gibbs energy for cavity formation becomes more positive. The $k_{\beta}$ values become more negative with decreasing cationic size in salt systems with a common anion, which indicates that the attractive forces between $\mathrm{NH}_{3}$ and cations become stronger.

The predicted $k_{s}$ values in the bromide and nitrate salt solutions are in reasonable agreement with the observed ones, if it is taken into account that since the experimental $k_{s}$ values at $c \rightarrow 0$ have been determined with the data at relatively high salt concentrations, they may have rather high uncertainties. Although the discrepancies between the predicted and observed $k_{s}$ values in the iodide salt solutions are rather large, at least the sign of $k_{s}$ values is consistent with that of the observed ones except the NaI system. ${ }^{s}$ The perchlorate salt systems yield the poorest agreement between the predicted and observed $k_{s}$ values. From these findings it may be said that the agreement becomes poorer with increasing ionic size. Masterton and Lee(7) pointed out that the $k$ values calculated from the scaled particle theory are very sensitive to ionic size and the effect is particularly serious with large ions, and that with smaller ions crystal radii can be used for satisfactory values of $\sigma_{3}$ and $\sigma_{4}$. If their indication is taken into account, it may be said that part of the large deviations in the perchlorate solutions is due to the employment of the crystal radius for $\sigma_{4}$. On the other hand, the good agreement in the nitrate solutions may be partly ascribed to the fact that the $\sigma_{4}$ value for the nitrate ion used in the calculations has been determined so as to $f i t$ the predicted $k_{s}$ values to the observed ones for nonpolar solutes in the alkali nitrate media(16). Thus, it may be necessary to determine $\sigma_{4}$ values for large anions by a method similar to that employed for the nitrate ion in order to get good agreement with experiment. Unfortunately, $k_{s}$ values for nonpolar solutes in perchlorate salt solutions do not seem to have been reported.

In conclusion, in view of various assumptions introduced in the theoretical evaluation and uncertainties of observed $k$ values, the estimated and observed Setchenow coefficients of ammonia are in taixly good agreement in the alkali bromide and nitrate solutions, which suggests the applicability of the present procedures as have been made on the basis of the scaled particle theory to the prediction of $k$ values for polar nonelectrolytes in aqueous solutions of salts with small ionic sizes.

\section{REFERENCES}

1) M. Randall and C. F. Failey, Chem. Rev., 4, 271 (1927).

2) F. A. Long and W. F. McDevit, Chem. Rev, ,51, 119 (1952).

3) B. E. Conway, "Ionic Hydration in Chemistry and Biophysics", Elsevier, Amsterdam, Chapter 20 (1981).

4) R. A. Pierotti, J. Phys. Chem., 67, 1840 (1963).

5) R. A. Pierotti, J. Phys. Chem., 69, 281 (1965).

6) S. K. Shoor and K. E. Gubbins, J. Phys. Chem., 73, 498 (1969).

7) W. L. Masterton and T. P. Lee, J. Phys. Chem., 74, 1776 (1970).

8) W. L. Masterton, J. Solution Chem., 4, 523 (1975).

9) M. Maeda, G. Nakagawa, and G. Biedermann, J. Phys. Chem., 87, 121 (1983).

10) M. Maeda, J. Phys. Chem., 90, 1134 (1986).

11) M. Maeda, J. Electrochem Soc. Jpn. (Denki Kagaku), 55, 61 (1987).

12) M. Maeda, M. Hayashi, S. Ikeda, Y. Kinjo, and K. I to, Bull. Chem. Soc. Jpn., $60,2047(1987)$.

13) $\bar{J}$. O. Hirschfelder, C. F. Curtis, and R. B. Bird, "Molecular Theory of Gases and Liquids", John Wiley \& Sons, New York, Chapter 3 (1954).

14) H. L. Friedman and C. V. Krishnan, "Water A Comprehensive Treatise", Franks Ed., Plenum, New York, Vol.3, Chapter 1 (1973).

15) T. C. Waddington, Trans. Faraday Soc., 62, 1482 (1966).

16) W. L. Masterton, D. Bolocofsky, and T. P. Lee, J. Phys. Chem., 75,2809 (1971).

17) Gmelins Handbuch der Anorganischen Chemie, "Chlor" Part B, Verlag Chemie, Weinheim (1969).

18) C. Mavroyannis ans M. J. Stephen, Mol. Phys., 5, 629 (1962).

19) B. E. Conway, "Electrochemical Data", Elsevier, New York (1952).

20) Landort-Börnstein, "Zahlen Werte und Funktion aus Physik-Chemie-AstronomieGeophysik-Technik", Vol.1, part 1 (1950). 